[Article]

\title{
光诱导约束刻蚀体系中羟基自由基生成的影响因素
}

\author{
胡 艳 方秋艳 周剑章* 詹东平 时 康 田中群 田昭武
}

(厦门大学化学化工学院化学系, 固体表面化学国家重点实验室, 福建厦门 361005)

摘要: 采用荧光分析, 暂态光电流响应分析, 电化学交流阻抗谱(EIS)和 Mott-Schottky 响应分析考察了外加电 位, 光照时间, 溶液 $\mathrm{pH}$ 等几个关键因素对光诱导约束刻蚀体系中 $\mathrm{TiO}_{2}$ 纳米管阵列表面游离 $\cdot \mathrm{OH}$ 生成的影响. 结果表明: 当外加电位为 $1.0 \mathrm{~V}$ 时, 光电协同产生游离 $\cdot \mathrm{OH}$ 效率最高; $\cdot \mathrm{OH}$ 的光催化生成与消耗能很快达到稳 态, 形成稳定的约束刻蚀剂层, 有利于保持刻蚀过程中的精度; 当 $\mathrm{pH}$ 为 10 时, $\mathrm{TiO}_{2}$ 纳米管光催化产生游离· $\mathrm{OH}$ 效率最高. 研究结果对于调控和优化光诱导约束刻蚀平坦化铜的溶液体系, 提高铜的刻蚀速度或平坦化精度有 重要的指导意义.

关键词：光诱导约束刻蚀; 游离 $\cdot \mathrm{OH}$; 苂光检测; 光电协同效应; $\mathrm{TiO}_{2}$ 纳米管阵列 中图分类号: 0649

\section{Factors Influencing Hydroxyl Radical Formation in a Photo-Induced Confined Etching System}

\author{
HU Yan FANG Qiu-Yan ZHOU Jian-Zhang* ZHAN Dong-Ping \\ SHI Kang TIAN Zhong-Qun TIAN Zhao-Wu
}

(State Key Laboratory of Physical Chemistry of Solid Surface, Department of Chemistry, College of Chemistry and Chemical Engineering, Xiamen University, Xiamen 361005, Fujian Province, P. R. China)

\begin{abstract}
In this paper, we studied the formation of free $\cdot \mathrm{OH}$ on a $\mathrm{TiO}_{2}$ nanotube array electrode in a photo-induced confined etching system. We used fluorescence spectroscopy, transient photocurrent response, electrochemical impedance spectroscopy (EIS), and Mott-Schottky analysis to investigate the influence of several key factors, including the applied potential, the illumination time, and the $\mathrm{pH}$ value. The highest efficiency for the photoelectrocatalytic formation of free $\cdot \mathrm{OH}$ on the $\mathrm{TiO}_{2}$ nanotube array electrode was achieved at an applied potential of $1.0 \mathrm{~V}$ ( $v$ s a saturated calomel electrode (SCE)); the photoelectrocatalytic generation and consumption of free $\cdot \mathrm{OH}$ quickly approached a steady state in this system, as the confined etching layer formed by $\cdot \mathrm{OH}$ remained stable during illumination. This may allow good control of the etching precision during continuous etching processes. The highest efficiency for the photoelectrocatalytic formation of free $\cdot \mathrm{OH}$ on the $\mathrm{TiO}_{2}$ nanotube array electrode was observed at $\mathrm{pH} 10$. The results have an important significance for regulating and optimizing photo-induced confined etching system, which can be used to improve the etching speed or the leveling precision during the planarization of copper.
\end{abstract}

Key Words: Photo-induced confined etching; Free $\cdot \mathrm{OH}$; Fluorescence detection;

Photo-electro-synergistic effect; $\mathrm{TiO}_{2}$ nanotube array

Received: July 9, 2013; Revised: September 4, 2013; Published on Web: September 4, 2013.

"Corresponding author. Email: jzzhou@xmu.edu.cn; Tel: +86-592-2189663.

The project was supported by the National Natural Science Foundation of China $(91023043,21021002,91023006)$.

国家自然科学基金(91023043, 21021002, 91023006)资助项目

(C) Editorial office of Acta Physico-Chimica Sinica 


\section{1 引言}

$\mathrm{TiO}_{2}$ 因光催化活性高, 价廉无毒, 稳定性好, 在 光催化降解污染物和染料敏化太阳能电池等方面 受到广泛关注. $\mathrm{TiO}_{2}$ 的光催化性能主要取决于光催 化生成的活性氧物种, 如羟基自由基 $(\cdot \mathrm{OH})$ 、超氧自 由基 $\left(\mathrm{O}_{2}^{-}\right)$和过氧化氢 $\left(\mathrm{H}_{2} \mathrm{O}_{2}\right)$, 其中 $\cdot \mathrm{OH}$ 则是光催化 氧化反应的主要物种. ${ }^{1-3} \cdot \mathrm{OH}$ 能够无选择性地氧化 多种有机物并且反应速率较快, 常用于环境工程中 降解污染物. ${ }^{4-7}$ 此外 $\cdot \mathrm{OH}$ 还可用于材料的抛光整平. Murata 等 ${ }^{8}$ 通过 Fenton 反应产生 $\cdot \mathrm{OH}$ 并将其应用于 刻蚀平坦化半导体 $\mathrm{GaN}(0001)$, 可以得到无划痕和 无腐蚀点的光滑表面. Hara 等9 研究了 CMP 装置中 通过工具 $\mathrm{Fe}$ 盘分解 $\mathrm{H}_{2} \mathrm{O}_{2}$ 产生 $\cdot \mathrm{OH}$ 用于刻蚀平坦化 半导体 $4 \mathrm{H}-\mathrm{SiC}(0001)$, 结果表明该方法可改善半导 体的表面粗糙度和表面晶型, 得到一个阶梯(stepterrace)结构的平整表面. Scholz 等 ${ }^{10}$ 简单地将 $\mathrm{Au}$ 表 面浸入 Fenton 试剂中, 利用产生的· $\mathrm{OH}$ 刻蚀氧化达 到平坦化的目的, 他们认为 $\cdot \mathrm{OH}$ 会优先氧化突起部 分然后逐渐地达到有效抛光的效果. 但目前还未有 文献报道以 $\mathrm{TiO}_{2}$ 为光催化剂产生 $\cdot \mathrm{OH}$ 用于材料表 面的平坦化.

近来我们课题组在约束刻蚀剂层技术 $(\mathrm{CELT})^{11}$ 的基础上提出了一种新的化学平坦化方法一一光 诱导约束刻蚀平坦化用于铜的刻蚀整平. ${ }^{2}$ 其原理 是: 在工具表面 $\left(\mathrm{TiO}_{2}\right.$ 纳米管阵列)利用光催化反应 产生刻蚀剂, 当刻蚀剂向被加工的工件表面扩散的 过程中与溶液中已存在的约束剂(本身没有刻蚀能 力)发生化学反应, 致使刻蚀剂的扩散受限, 被约束 在紧靠模板工具表面薄层内, 即形成了约束刻蚀剂 层, 当被加工工件进入约束刻蚀剂层后发生刻蚀并 去除, 最终获得与工具面形精度相当的平面. 约束 刻蚀剂层厚度及浓度分布决定了刻蚀的精度和速 度, 其产生和控制是该平坦化方法的核心问题. $\mathrm{TiO}_{2}$ 光催化产生的 $\mathrm{OH}$ 以两种形式存在: 吸附态 的 $\cdot \mathrm{OH}$ 和游离态的 $\cdot \mathrm{OH}$. 它们在光催化降解中均能 发挥作用, 而在约束刻蚀体系中则大为不同, 只有 游离态的 $\cdot \mathrm{OH}$ 可以扩散到工件表面对其进行刻蚀. - $\mathrm{OH}$ 作为刻蚀剂, 其浓度既决定了刻蚀的速度, 又 会影响约束刻蚀剂层的厚度进而影响刻蚀的精度. 因此, 探讨 $\mathrm{TiO}_{2}$ 纳米管阵列表面游离. $\mathrm{OH}$ 的光催化 生成及影响因素, 对指导 $\mathrm{Cu}$ 的约束刻蚀平坦化非常 重要. 本文以禁带宽度为 $3.2 \mathrm{eV}$ 的锐钛矿型 $\mathrm{TiO}_{2}$ 纳 米管阵列为光催化剂, 在光诱导下生成 $\mathrm{OH}$, 采用荧
光分析法检测游离 $\cdot \mathrm{OH}$ 的浓度, ${ }^{1,2}$ 通过暂态光电流 响应分析, 电化学交流阻抗谱和 Mott-Schottky 响应 分析考察外加电位, 光照时间, 溶液 $\mathrm{pH}$ 等因素对 $\mathrm{TiO}_{2}$ 光催化产生游离 $\cdot \mathrm{OH}$ 的影响, 调控和优化用于 光诱导约束刻蚀平坦化铜的溶液体系, 提高铜的刻 蚀平坦化精度.

\section{2 实验部分}

\section{1 试剂与仪器}

试剂: 对苯二甲酸(纯度 $99 \%$, 国药集团化学试 剂有限公司); 氢氧化钠(分析纯, 广州化学试剂分公 司); $\mathrm{NH}_{4} \mathrm{~F}$ 、丙三醇、嗍酸、无水乙醇、丙酮(分析纯, 国 药集团化学试剂有限公司); $\mathrm{Ti}$ 管(纯度 $99.6 \%$, 宝鸡 宏顺达钛业有限公司); 氮气(高纯, 99.999\%, 厦门林 德气体有限公司).

仪器: 光照光源采用北京赛凡光电的 $500 \mathrm{~W}$ 氙 灯; 荧光测试采用日本 Hitachi 公司的 F-7000 型荧光 光谱仪; 暂态光电流测试的光源采用北京卓立汉光 生产的 $150 \mathrm{~W}$ 氙灯, 测量光电流的恒电位仪采用上 海辰华仪器有限公司的 CHI630a; 电化学阻抗谱测 试采用美国 EG\&G 的 PAR273 恒电位仪及 5206 Two Phase Lock-in Amplifier 锁相放大器; 电镜测试 采用日本 Hitachi 公司的 S4800 型扫描电镜.

\section{$2.2 \mathrm{TiO}_{2}$ 纳米管阵列光催化剂的制备}

将 $\mathrm{Ti}$ 箔(长、宽、厚分别为 $10 、 15 、 1 \mathrm{~mm}$ ) 依次用 丙酮、无水乙醇、超纯水超声清洗两次, 每次 10-15 $\min$. 然后用氮气吹干备用. 制备装置采用两电极体 系, 以 $\mathrm{Ti}$ 䇴为阳极, 铂片为阴极, 含 $\mathrm{NH}_{4} \mathrm{~F}$ 浓度为 $0.5 \%(w)$ 的 $\mathrm{NH}_{4} \mathrm{~F}$-丙三醇为电解液, 在 $20 \mathrm{~V}$ 的电压 下阳极氧化 $30 \mathrm{~min}$. 然后在 $450{ }^{\circ} \mathrm{C}$ 下退火 $2 \mathrm{~h}$ 使 $\mathrm{TiO}_{2}$ 由无定形转化为锐钛矿晶型. 由 SEM 图(见 Supporting Information)可以看出样品表面呈现出规整、高 度有序的 $\mathrm{TiO}_{2}$ 纳米管阵列结构. 所制备的 $\mathrm{TiO}_{2}$ 管径 约为 $100 \mathrm{~nm}$, 壁厚约为 $8 \mathrm{~nm}$. 阳极氧化法制备的 $\mathrm{TiO}_{2}$ 是垂直基底有序生长的, 有利于自由电子向基 底的移动, 同时纳米管阵列的中空结构增大了其与 溶液的接触. ${ }^{13-15}$ 与光催化常用的 $\mathrm{TiO}_{2}$ 纳米微粒膜相 比, $\mathrm{TiO}_{2}$ 纳米管虽然在比表面积上不占优势, 但其具 有较短的载流子扩散路径, 极大地降低了光生载流 子的复合, 在很大程度上弥补了其比表面较小的不 足. ${ }^{16}$ 同时, 与纳米微粒膜相比, 阳极氧化法制备的 $\mathrm{TiO}_{2}$ 纳米管阵列具有更为平整的表面, 在约束刻蚀 体系中有利于形成较为平整的刻蚀剂层. 


\section{3 实验条件及参数}

本实验中荧光测试以上述方法制备的 $\mathrm{TiO}_{2}$ 纳 米管阵列为光催化剂, 在自制的光电解池中用白光 光照 $15 \mathrm{~min}$ (光强为 $1.58 \mathrm{~W} \cdot \mathrm{cm}^{-2}$ ), 溶液为 $1 \mathrm{mmol}$. $\mathrm{L}^{-1}$ 的对苯二甲酸 (TA) 和 $0.01 \mathrm{~mol} \cdot \mathrm{L}^{-1}$ 的 $\mathrm{NaOH}$, 体积 为 $3 \mathrm{~mL}$. 光照结束后采用苂光光谱仪测定产物 2-羟 基对苯二甲酸(TAOH)的苂光强度. 文中实验均是在 未除氧下进行, 实验过程中保持光强不变, 光源与 电极, 辅助电极与工作电极间距离恒定, 仅改变单 一变量如外加电位, 光照时间, 溶液 $\mathrm{pH}$. 荧光测试的 主要参数如下, 激发波长为 $312 \mathrm{~nm}$, 激发和发射的 狭缝宽度均为 $2.5 \mathrm{~nm}$, 光电倍增管 (PMT) 电压为 $700 \mathrm{~V}$. 暂态光电流及电化学阻抗谱测试(测试频率 $100 \mathrm{kHz}-100 \mathrm{mHz}$ )均以 $\mathrm{TiO}_{2}$ 光电极为工作电极, $\mathrm{Pt}$ 丝为对电极, 饱和甘录电极 (SCE) 为参比电极, 测试 体系为 $0.01 \mathrm{~mol} \cdot \mathrm{L}^{-1} \mathrm{NaOH}$ 溶液, 测试光强为 35 $\mathrm{mW} \cdot \mathrm{cm}^{-2}$. 另外, 本文中所有电位均是相对于饱和 甘录电极.

\section{3 结果与讨论}

由于在光诱导约束刻蚀 $\mathrm{Cu}$ 体系中, 实现约束刻 蚀 $\mathrm{Cu}$ 的是游离态的 $\cdot \mathrm{OH}$. 因此, 不能采用文献中的 诸如降解有机物等 $\mathrm{TiO}_{2}$ 光催化效率的评估方法来 表征· $\mathrm{OH}$ 的生成. 我们选择荧光分析法对实验过程 中游离的 $\cdot \mathrm{OH}$ 进行定量检测. ${ }^{2}, 17$ 以无苂光特性的对 苯二甲酸为分子探针, 它与 $\cdot \mathrm{OH}$ 反应后生成专一稳 定且具有很强苂光特性的 2-羟基对苯二甲酸产物, 速率常数 $k_{1}=3.3 \times 10^{9} \mathrm{~L} \cdot \mathrm{mol}^{-1} \cdot \mathrm{s}^{-1}$. ${ }^{18}$ 通过测量 $424 \mathrm{~nm}$ 处产物 TAOH 的苂光峰强度可间接测出. $\mathrm{OH}$ 的浓 度. 反应式见示意图 1.

\section{1 外加电位对羟基自由基生成的影响}

外加电位能改变 $\mathrm{TiO}_{2}$ 电极的费米能级及本体 内能带的弯曲量. 当施加合适的外加电位使 $\mathrm{TiO}_{2}$ 的 能带弯曲增大, 这将有利于光生电子导入外电路和 空穴移至 $\mathrm{TiO}_{2}$ 表面, 减少载流子的无效复合, 从而

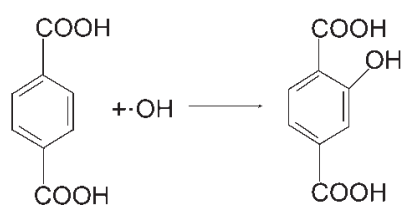

示意图 1 荧光检测· OH 的反应式

Scheme 1 Chemical equation of fluorescence detection of $\cdot \mathrm{OH}$

No fluorescent signal detected for the reactant (TA), while the product $(\mathrm{TAOH})$ emits a strong fluorescence signal.
能大大提高光催化效率. 光照的同时在 $\mathrm{TiO}_{2}$ 电极上 施加一定的外加电位, 即光电协同催化 $\mathrm{TiO}_{2}$ 用于降 解有机污染物已有报道. 孙岗等 ${ }^{19}$ 提到施加 $0.6 \mathrm{~V}$ 外 加电位时光电催化降解苯酚的性能最好; Yon 等 ${ }^{20}$ 报 道施加 $0.1 \mathrm{~V}$ 的较小外加电位时降解甲基橙的效率 已经足够; Philippidis 等 ${ }^{21}$ 报道当施加 $1.5 \mathrm{~V}$ 的外加 电位, 光电协同催化降解吡虫啉的表观速率常数与 光催化相比提高了 $249 \%$. 然而, 光催化降解体系与 我们的光诱导约束刻蚀体系存在前述的 $\cdot \mathrm{OH}$ 作用 机制的差别, 因此, 有必要采用另外的方法重新评 估外加电位对 $\mathrm{TiO}_{2}$ 光催化生成· $\mathrm{OH}$ 的影响. 本文选 用苂光分析法检测不同电位下生成的游离 $\cdot \mathrm{OH}$ 浓 度大小. 图 1 为 $\mathrm{TAOH}$ 在不同外加电位下的苂光光 谱图及 $424 \mathrm{~nm}$ 处的荧光强度与外加电位的关系曲 线. 由图 1 可知, 随着外加电位的增大, 苂光强度逐 渐增大, 当外加电位增加到 $1.0 \mathrm{~V}$ 附近时, 荧光强度
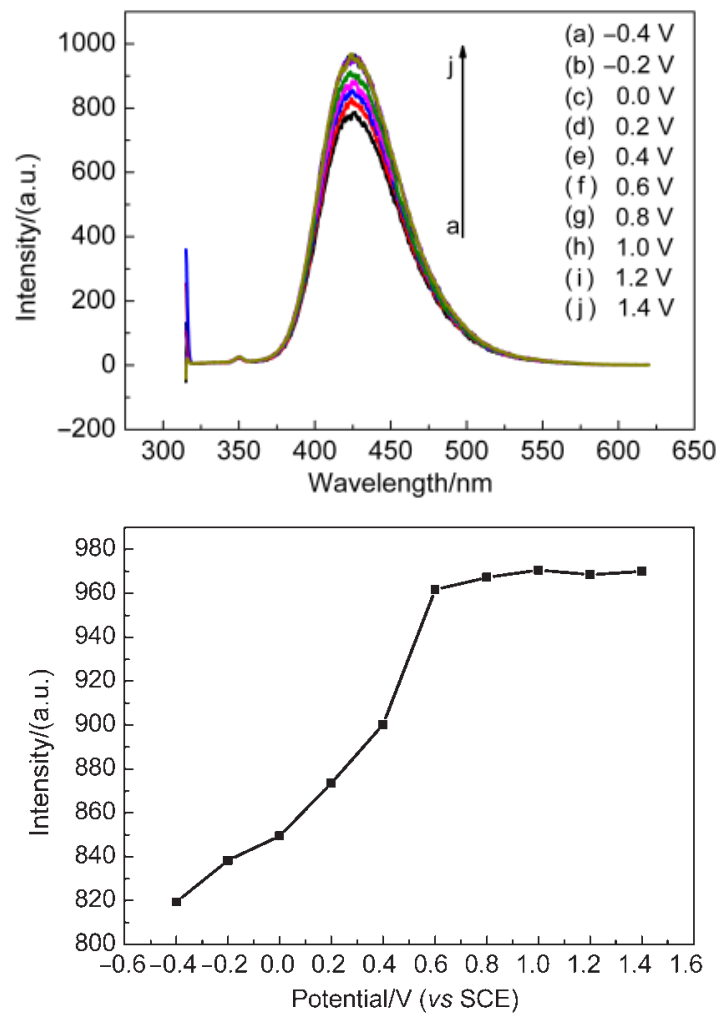

图 1 (a) TAOH 在不同外加电位下的荧光光谱图及 (b) $424 \mathrm{~nm}$ 处的荧光强度与外加电位的关系曲线

Fig.1 (a) Fluorescence spectra of TAOH at different applied potentials and (b) effect of applied potentials on the induced fluorescence intensity of TAOH at $424 \mathbf{n m}$ The fluorescence intensity at $424 \mathrm{~nm}$ is the fluorescence peak of $\mathrm{TAOH}$, which can indirectly represent the concentration of free $\cdot \mathrm{OH}$, the initial solution consisting of $1 \mathrm{mmol} \cdot \mathrm{L}^{-1} \mathrm{TA}$, and $0.01 \mathrm{~mol} \cdot \mathrm{L}^{-1} \mathrm{NaOH}$. volume of the solution: $3 \mathrm{~mL}$, light intensity: $1.58 \mathrm{~W} \cdot \mathrm{cm}^{-2}$, illumination time: $15 \mathrm{~min}$ 


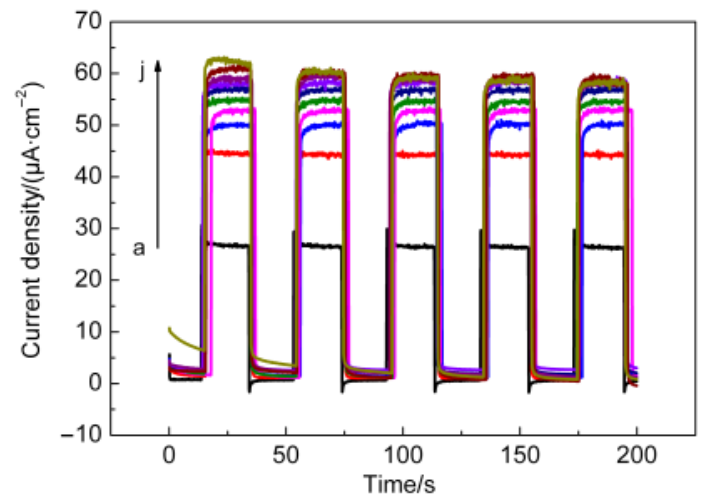

图 2 不同外加偏压下 $\mathrm{TiO}_{2}$ 纳米管阵列的暂态光电流响应

Fig.2 Transient photocurrent responses of $\mathrm{TiO}_{2}$ nanotube arrays at different applied potentials white light illumination, light intensity: $35 \mathrm{~mW} \cdot \mathrm{cm}^{-2}$; applied potential $/ \mathrm{V}$ : (a) -0.4 , (b) -0.2 , (c) 0 , (d) 0.2 , (e) 0.4 , (f) 0.6, (g) 0.8 , (h) 1 , (i) 1.5 , (j) 2

变化趋于平缓. 这表明当外加电位达到 $1.0 \mathrm{~V}$ 附近 时, 羟基自由基的生成量趋于稳定, 此时继续增大 电位对羟基自由基的生成量贡献不大.

\subsection{1 不同外加电位下 $\mathrm{TiO}_{2}$ 纳米管的暂态光电流 响应}

为了更好地了解外加电位对 $\mathrm{TiO}_{2}$ 纳米管光电 性能的影响, 实验测试了不同外加电位 $(-0.4-2.0$ $\mathrm{V}(v S \mathrm{SCE}))$ 下的暂态光电流响应. 如图 2 所示, 当外 加电位从 $-0.4 \mathrm{~V}$ 升至 $1.0 \mathrm{~V}$ 时, 随着外加电位增大, 光电流逐渐增大, 当外加电位增至 $1.0 \mathrm{~V}$ 后, 光电流 的变化趋于平缓. 由于外加电位能使 $\mathrm{TiO}_{2}$ 的能带弯 曲增大, 减少了电子与空穴的复合, 所以随着外加 电位的增大, 光电响应显著增强. 然而由于 $\mathrm{TiO}_{2}$ 阵 列膜的厚度是有限的, 空间电荷层的厚度最大不能 超过半导体氧化膜的厚度. 且当光强固定时, 光生 电子的数量是一定的, 故外加电位达到一定值时, 光生载流子已达到充分分离, 形成了饱和光电流. 此时继续增加电位对光电流的提高幅度不大. $\mathrm{TiO}_{2}$ 阳极光电流的变化可以反映空穴和· $\mathrm{OH}$ 生成速率 的变化, 光电流的增加表明了 $\cdot \mathrm{OH}$ 的产生速率增 大. ${ }^{19}$ 也就是说外加电位为 $1.0 \mathrm{~V}$ 左右时, $\cdot \mathrm{OH}$ 的产 生速率达到最大. 这变化趋势与不同电位下的菼光 光谱是相符的.

\subsubsection{EIS 及 Mott-Schottky 测试}

电化学交流阻抗谱(EIS) 是一种频率域测量方 法, 可获得电极界面的动力学信息, 通过 EIS 谱可揭 示光生载流子的传输特性同半导体材料的光催化 活性之间的关系, 而 Mott-Schottky 测试则能进一步
得出半导体的平带电位、载流子浓度等特征参数. 如图 3 所示, 光照下随着外加电位减小, 阻抗圆弧逐 渐变大. 因为 Nyquist图中的阻抗曲线只有一个半圆 弧, 说明在测量频率范围 $(100 \mathrm{kHz}-100 \mathrm{mHz})$ 内, $\mathrm{TiO}_{2}$ 的光电化学反应只有一个速率控制步骤, 即电 荷转移过程. 阻抗弧半径越小, 则阻抗值也越小, 即 电荷转移电阻越小. 已有的研究表明, ${ }^{22-25}$ Nyquist图 上的圆弧半径对应着电荷转移电阻和光生电子-空 穴对的分离效率, 圆弧半径越小, 光催化反应进行 得越快. 在光照条件下, 随着外加电位增大, 阻抗圆 弧半径减小, 光催化反应加快, $\mathrm{TiO}_{2}$ 纳米管阵列表面 产生的大量光生电子被导入外电路, 有利于光生电 子-空穴的分离, 使得有更多的空穴可输运到电极/
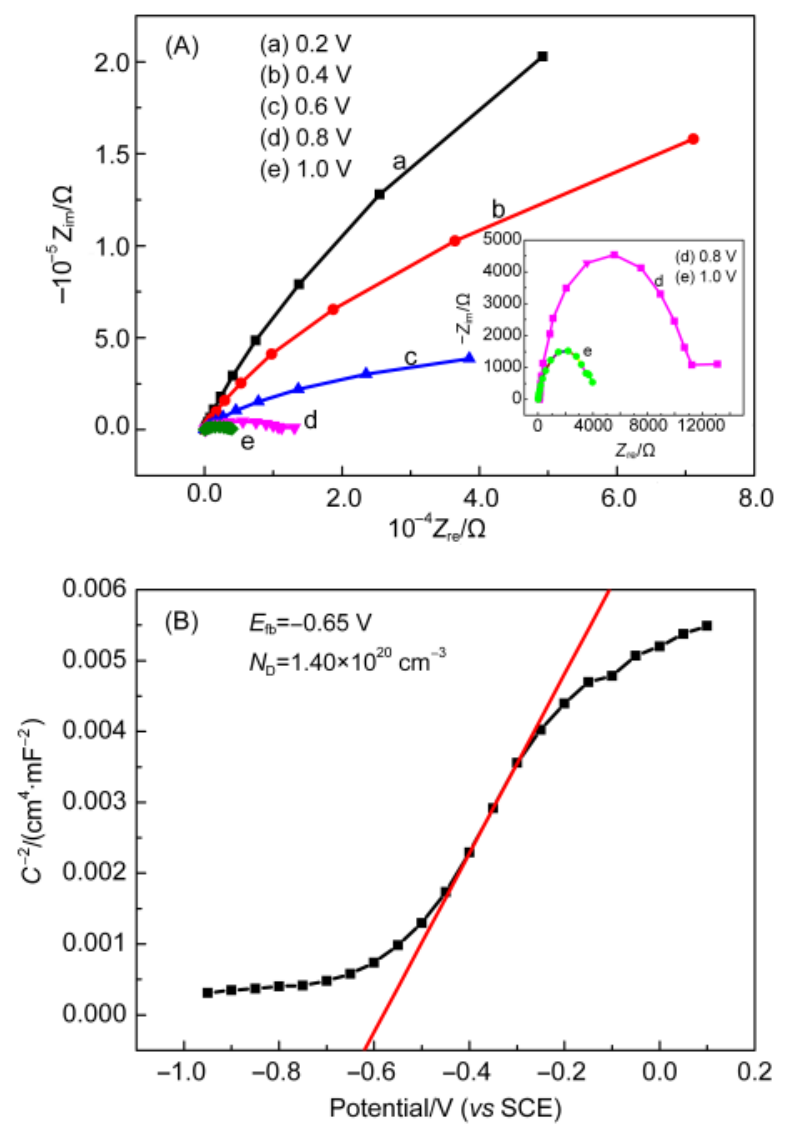

图 3 光照下 $0.01 \mathrm{~mol} \cdot \mathrm{L}^{-1} \mathrm{NaOH}$ 溶液中(a)不同外加 电位下 $\mathrm{TiO}_{2}$ 纳米管阵列电极的 Nyquist 图及(b) $\mathrm{TiO}_{2}$ 纳米管阵列电极的 Mott-Schottky 曲线

Fig.3 (a) Nyquist impedence plots of $\mathrm{TiO}_{2}$ nanotube arrays at different applied potentials and (b) Mott-Schottky curves of $\mathrm{TiO}_{2}$ nanotube arrays under illumination in $0.01 \mathrm{~mol} \cdot \mathrm{L}^{-1} \mathrm{NaOH}$ solution

(A) The frequencies are in the range of $100 \mathrm{kHz}-100 \mathrm{mHz}$ with light intensity kept at $35 \mathrm{~mW} \cdot \mathrm{cm}^{-2}$. (B) The light intensity is kept at $35 \mathrm{~mW} \cdot \mathrm{cm}^{-2}$. 
溶液界面处氧化 $\mathrm{H}_{2} \mathrm{O}$ 或 $\mathrm{OH}^{-}$产生・ $\mathrm{OH}$. 根据 MottSchottky 公式:26

$$
C_{\mathrm{sc}}^{-2}=2\left(E-E_{\mathrm{fb}}-k T / e\right) /\left(\varepsilon_{\mathrm{sc}} \varepsilon_{0} e N_{\mathrm{D}}\right)
$$

其中, $C_{\mathrm{sc}}$ 为半导体空间电荷层的电容, $E$ 为电极电 位, $E_{\mathrm{fb}}$ 为平带电位, $\varepsilon_{0}$ 为真空介电常数, $\varepsilon_{0}=8.85 \times 10^{-12}$ $\mathrm{F} \cdot \mathrm{m}^{-1}, \varepsilon_{\mathrm{sc}}$ 为半导体空间电荷层的相对介电常数, $\varepsilon_{\mathrm{sc}}=$ 10 , 由图中线性部分的斜率和截距可求得 $\mathrm{TiO}_{2}$ 的掺 杂浓度和平带电位, 载流子浓度 $N_{\mathrm{D}}=1.40 \times 10^{20} \mathrm{~cm}^{-3}$, $E_{\mathrm{fb}}$ 约为 $-0.65 \mathrm{~V}$. 根据公式

$$
d_{\mathrm{sc}}=\left\{2 \varepsilon \varepsilon_{0}\left(E-E_{\mathrm{FB}}\right) /\left(q N_{\mathrm{D}}\right)\right\}^{1 / 2}
$$

可进一步求得当施加 $1.0 \mathrm{~V}$ 的外加电位时, $\mathrm{TiO}_{2}$ 半导 体的空间电荷层厚度约为 $3.6 \mathrm{~nm}$. 对于中空的 $\mathrm{TiO}_{2}$ 纳米管, 两侧空间电荷层的厚度相加应为该值的两 倍, ${ }^{16}$ 整个空间电荷层的厚度约为 $7.2 \mathrm{~nm}$, 这一值与 $\mathrm{SEM}$ 数据中的 $\mathrm{TiO}_{2}$ 纳米管的壁厚(约为 $8 \mathrm{~nm}$ ) 接近. 根据冷文华等 ${ }^{27}$ 报道, 当空间电荷层的厚度等于 $\mathrm{TiO}_{2}$ 的膜层厚度时, $\mathrm{TiO}_{2}$ 的光催化效率最高. 在我们 的体系中, 当外加电位达到 $1.0 \mathrm{~V}$ 时, 整个纳米管的 管壁全部构成了空间电荷层, 此时, $\mathrm{TiO}_{2}$ 纳米管的光 催化效率达到最大.

3.1.3 $\mathrm{TiO}_{2}$ 纳米管光催化生成 $\cdot \mathrm{OH}$ 中的光电协同 作用

一般情况下, $\mathrm{TiO}_{2}$ 光催化生成 $\cdot \mathrm{OH}$ 的原理: 当 入射光的能量大于 $\mathrm{TiO}_{2}$ 的 $E_{\mathrm{g}}$ 时, 在耗尽层内建电场 的作用下, 光生电子-空穴分离. 空穴移至 $\mathrm{TiO}_{2}$ 表面 时与其表面吸附的 $\mathrm{H}_{2} \mathrm{O}$ 或 $\mathrm{OH}^{-}$反应生成 $\cdot \mathrm{OH}$. 但其 光生电子-空穴的复合几率往往较大. 当光与电协同 作用时, 适当的外加电位可使 $\mathrm{TiO}_{2}$ 能带弯曲增大, 空间电荷层压降增大, 有利于光生电子运动至电极 内部进而导入外电路, 而光生空穴则移至 $\mathrm{TiO}_{2}$ 表 面, 避免了光生电子-空穴的简单复合; 同时, 通过控 制外加电位还可调控 $\mathrm{TiO}_{2}$ 半导体空穴的准 Fermi 能 级与 $\mathrm{H}_{2} \mathrm{O} / \cdot \mathrm{OH}$ 的氧化还原电位匹配. 光照下, 施加 合适的外加电位, 即光电协同催化可以大大提高光 催化效率. 此外, 有报道发现, 在无光照条件下, 当 外加电位达到 $3.0 \mathrm{~V}(v \mathrm{Ag} / \mathrm{AgCl})$ 时, 可直接激发产 生电子-空穴对. ${ }^{28}$ 这主要是因为外加电位能调控 $\mathrm{TiO}_{2}$ 的 Fermi 能级, 外加电位的增大导致了 $\mathrm{TiO}_{2}$ 半 导体 Fermi 能级下移, 半导体的能带弯曲程度也跟 着不断增大. 当电位达到 $3.0 \mathrm{~V}(v s \mathrm{Ag} / \mathrm{AgCl})$ 时, Fer$\mathrm{mi}$ 能级与 $\mathrm{TiO}_{2}$ 价带位置持平, 价带的电子直接隧穿 到导带并在外电场的作用下导入到外电路, 过剩的 空穴则移至 $\mathrm{TiO}_{2}$ 表面, 空穴与表面吸附的 $\mathrm{H}_{2} \mathrm{O}$ 或
$\mathrm{OH}^{-}$反应生成· $\mathrm{OH}$. 但当电位超过 $1.5 \mathrm{~V}$ 时, $\mathrm{TiO}_{2}$ 电 极表面会发生析氧等副反应(见 Supporting Information). 在我们的光诱导约束刻蚀体系中, 副产物 $\mathrm{O}_{2}$ 会导致工具 $\mathrm{Cu}$ 表面的氧化, 较大的外加电位虽然可 能会增加 $\cdot \mathrm{OH}$ 的生成量, 但对我们的光诱导约束刻 蚀 $\mathrm{Cu}$ 体系是不利的. 另外, 氧还原对游离 $\cdot \mathrm{OH}$ 的生 成也有一定贡献, 这部分影响较为复杂. 且本文主 要侧重于考察外加电位等参数对于游离. $\mathrm{OH}$ 生成 的影响, 对游离· $\mathrm{OH}$ 生成机理暂不做深入探讨.

\section{2 光照时间对羟基自由基生成的影响}

约束刻蚀体系的化学平坦化精度虽然较高, 但 需要的刻蚀时间较长. 长时间持续光照下 $\mathrm{TiO}_{2}$ 纳米 管阵列光催化生成. $\mathrm{OH}$ 及被捕捉剂约束的复合过 程能否稳定, 进而保持一个稳定的约束刻蚀剂层是 保证平坦化精度的一个重要前提. 图 4(a) 是不同光 照时间下的苂光光谱图. 图中波长为 $424 \mathrm{~nm}$ 处出现 了 $\mathrm{TAOH}$ 的荧光特征峰, 并且随着光照时间的增加, 特征峰的强度不断增强, 表明溶液中的 TAOH 浓度
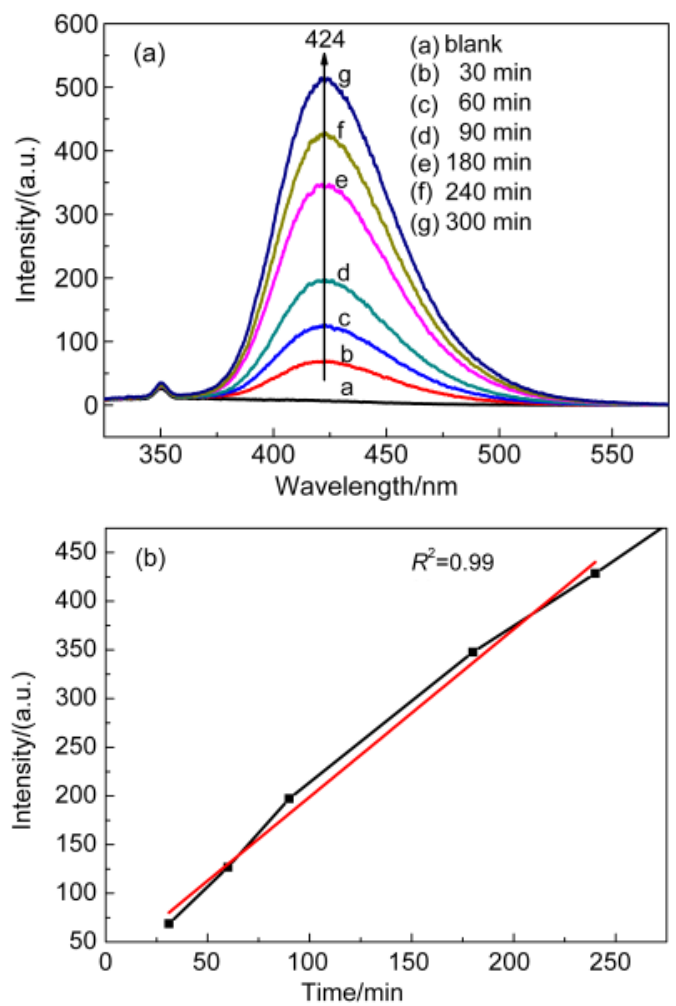

图 4 (a) 不同光照时间下的荧光光谱图及(b) $424 \mathrm{~nm}$ 处 荧光强度与光照时间关系图

Fig.4 (a) Fluorescence spectra of TAOH at different illumination time and (b) the induced fluorescence intensity of ТАОН versus light illumination time at $424 \mathbf{n m}$ the initial solution consisting of $1 \mathrm{mmol} \cdot \mathrm{L}^{-1} \mathrm{TA}$ and $0.01 \mathrm{~mol} \cdot \mathrm{L}^{-1}$ $\mathrm{NaOH}$, volume of the solution: $3 \mathrm{~mL}$, light intensity: $35 \mathrm{~mW} \cdot \mathrm{cm}^{-2}$ 
不断增加, 而 $\mathrm{TAOH}$ 的浓度与产生的· $\mathrm{OH}$ 的浓度成 一定的正比关系, 与文献 ${ }^{17}$ 的结果相一致. 因此, 424 $\mathrm{nm}$ 处的特征峰的强度间接反映了 $\cdot \mathrm{OH}$ 的生成量(实 验中表征的· $\mathrm{OH}$ 的生成量对应的是整个光照时间 $\cdot \mathrm{OH}$ 的累积生成量). 并且由图 4(b)中光照时间与波 长 $424 \mathrm{~nm}$ 处特征峰荧光强度的关系图可知, $\cdot \mathrm{OH}$ 的 生成量随反应时间的增加呈现较好的线性增加关 系, 其生成反应符合零级反应动力学规律, $\cdot \mathrm{OH}$ 的 单位时间生成速率是恒定的. 该体系中的 $\cdot \mathrm{OH}$ 的光 催化生成与被捕捉能很快就达到稳态, 因此其形成 的有效约束刻蚀剂层也能保持稳定, 不随光照时间 的变化而变化. 在实际应用于 $\mathrm{Cu}$ 平坦化的光诱导约 束刻蚀体系中, ${ }^{12}$ 我们采用甘氨酸(Gly)作为约束剂, $\cdot \mathrm{OH}$ 与 $\mathrm{TA}^{18} 、 \mathrm{Gly}^{29}$ 的反应速率常数在同一数量级, 可推测 $\cdot \mathrm{OH}$ 与 $\mathrm{Gly}$ 形成的约束刻蚀剂层能很快达到 稳定. 该结果也表明在一定的时间范围内光诱导约 束刻蚀体系用于刻蚀平坦化时, 随着光照持续, 约 束刻蚀剂层的厚度是稳定的, 不会因为其变化而影 响平坦化的精度.

\section{3 溶液 $\mathrm{pH}$ 值对羟基自由基生成的影响}

溶液 $\mathrm{pH}$ 值会改变 $\mathrm{TiO}_{2}$ 的能级位置及表面的荷 电状态, 是影响 $\mathrm{TiO}_{2}$ 光催化的参数之一. 文献 ${ }^{30,31}$ 报 道, 当 $\mathrm{pH} \approx 6.8$, 即为 $\mathrm{TiO}_{2}$ 的零电荷点 $(\mathrm{PZC})$ 时, $\mathrm{TiO}_{2}$ 表面能吸附最多的污染物, 所以其光催化性能在 $\mathrm{pH} \approx 6.8$ 时最高. 然而, 在我们的光诱导约束刻蚀体 系中, 不存在污染物在 $\mathrm{TiO}_{2}$ 表面的吸附问题, $\mathrm{pH}$ 的 改变只会影响 $\mathrm{TiO}_{2}$ 的能级位置及溶液中刻蚀剂前 驱体 $\left(\mathrm{OH}^{-}\right)$的浓度. 我们通过考察不同 $\mathrm{pH}$ 下光照后

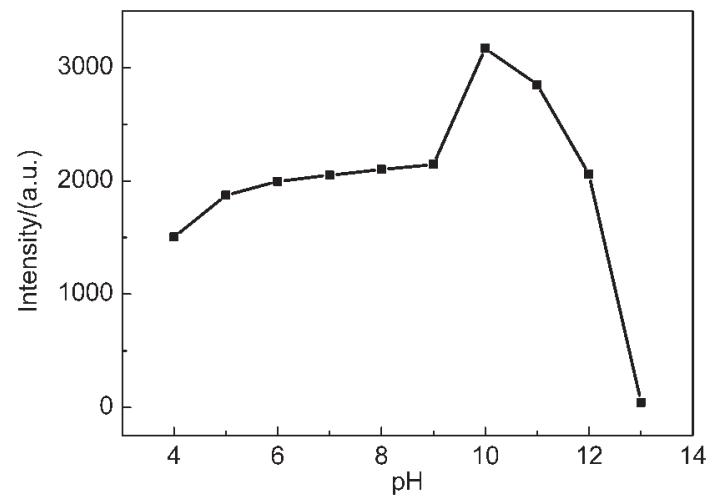

图 5 光照后溶液在 $424 \mathrm{~nm}$ 荧光谱峰强度与 pH 值的关系曲线

Fig.5 Effect of $\mathrm{pH}$ values on the fluorescence intensity at $424 \mathrm{~nm}$ in the photo-induced solution the initial solution consisting of $1 \mathrm{mmol} \cdot \mathrm{L}^{-1} \mathrm{TA}$ and $0.01 \mathrm{~mol} \cdot \mathrm{L}^{-1}$ $\mathrm{NaOH}$, volume of the solution: $3 \mathrm{~mL}$, light intensity: $1.58 \mathrm{~W} \cdot \mathrm{cm}^{-2}$, illumination time: $15 \mathrm{~min}$
溶液在 $424 \mathrm{~nm}$ 处的荧光特征峰的变化规律(以 TA 作为游离 $\cdot \mathrm{OH}$ 捕捉剂)来考察 $\mathrm{pH}$ 对约束刻蚀溶液体 系的影响. 如图 5 所示, 在本体系中 $\mathrm{TiO}_{2}$ 光催化产生 游离. $\mathrm{OH}$ 的反应受到 $\mathrm{pH}$ 的影响是非线性的. 在 $\mathrm{pH}$ 值 4 到 9 的区间, 随着 $\mathrm{pH}$ 的增大, 苂光强度缓慢增 加; $\mathrm{pH}$ 值大于 10 后荧光强度又随着 $\mathrm{pH}$ 的增大而急 剧减小; $\mathrm{pH}$ 值为 10 左右时, 荧光强度达到最大, 即 生成的游离态 $\cdot \mathrm{OH}$ 浓度最高. 因此, 本文其它实验 中所采用的 $\mathrm{pH}$ 值均为 10 .

\section{4 结 论}

本文采用苂光分析法结合暂态光电流响应, EIS 及 Mott-Schottky 测试考察了外加电位、光照时 间及 $\mathrm{pH}$ 值对光诱导约束刻蚀体系中 $\mathrm{TiO}_{2}$ 光催化产 生游离 $\cdot \mathrm{OH}$ 的影响. 结果表明:

(1) 光电协同作用于 $\mathrm{TiO}_{2}$ 纳米管, 可对游离态 - $\mathrm{OH}$ 的生成进行调控. 外加电位达到 $1.0 \mathrm{~V}$ 附近时, 游离· $\mathrm{OH}$ 的生成量趋于稳定, 继续增大电位对羟基 自由基的生成量贡献不大. 原因是当外加电位达到 $1.0 \mathrm{~V}$ 时, 整个纳米管管壁全部构成了空间电荷层, 此时, $\mathrm{TiO}_{2}$ 纳米管的光催化效率达到最大.

(2) $\cdot \mathrm{OH}$ 的生成量与光照反应时间呈现较好的 线性增加关系, 其生成反应符合零级反应动力学规 律. 该体系中的· $\mathrm{OH}$ 的光催化生成与消耗能很快达 到稳态, 其形成的约束刻蚀剂层能保持稳定.

(3) 在 $\mathrm{pH}$ 值 4 到 9 的区间内, 改变 $\mathrm{pH}$ 值对 $\mathrm{TiO}_{2}$ 纳米管光催化产生游离・ $\mathrm{OH}$ 影响不大, $\mathrm{pH}$ 值为 10 时, $\mathrm{TiO}_{2}$ 纳米管光催化产生游离 $\cdot \mathrm{OH}$ 的效率最高.

Supporting Information: available free of charge via the internet at http://www.whxb.pku.edu.cn.

\section{References}

(1) Ishibashi, K.; Fujishima, A.; Watanabe, T.; Hashimoto, K. J. Photochem. Photobiol. A 2000, 134, 139. doi: 10.1016/S10106030(00)00264-1

(2) Hirakawa, T.; Nosaka, Y. Langmuir 2002, 18, 3247. doi: 10.1021/la015685a

(3) Ishibashi, K.; Fujishima, A.; Watanabe, T.; Hashimoto, K. Electrochem. Commun. 2000, 2, 207. doi: 10.1016/S1388-2481 (00)00006-0

(4) Jorge, S. M. A.; Sene, J. J. D.; Florentino, A. D. O. J. Photochem. Photobiol. A 2005, 174, 71. doi: 10.1016/j. jphotochem.2005.03.010

(5) Ku, Y.; Lee, Y. C.; Wang, W. Y. J. Hazard. Mater. 2006, 138, 
350. doi: 10.1016/j.jhazmat.2006.05.057

(6) Macak, J. M.; Zlamal, M.; Krysa, J.; Schmuki, P. Small 2007, 3, 300.

(7) Philippidis, N.; Sotiropoulos, S.; Efstathiou, A.; Poulios, I. J. Photochem. Photobiol. A 2009, 204, 129. doi: 10.1016/j. jphotochem.2009.03.007

(8) Murata, J.; Sadakuni, S.; Yagi, K.; Sano, Y.; Okanoto, T.; Arima, K.; Hattori, A. N.; Mimura, H.; Yamauchi, K. Jpn. J. Appl. Phys. 2009, 48, 121001. doi: 10.1143/JJAP.48.121001

(9) Yagi, K.; Murata, J.; Kubota, A.; Sano, Y.; Hara, H.; Arima, K.; Okamoto, T.; Mimura, H.; Yamauchi, K. Jpn. J. Appl. Phys. 2008, 47, 104. doi: 10.1143/JJAP.47.104

(10) Nowicka, A. M.; Hasse, U.; Hermes, M.; Scholz, F. Angew. Chem. Int. Edit. 2010, 49, 1061. doi: 10.1002/anie.v49:6

(11) Tian, Z. W.; Fen, Z. D.; Tian, Z. Q.; Zhuo, X. D.; Mu, J. Q.; Li, C. Z.; Lin, H. S.; Ren, B.; Xie, Z. X.; Hu, W. Faraday Discuss. 1992, 94, 37. doi: 10.1039/fd9929400037

(12) Fang, Q. Y.; Zhou, J. Z.; Zhan, D. P.; Shi, K.; Tian, Z. W.; Tian, Z. Q. Chem. Commun. 2013, 49, 6451 doi: 10.1039/c3cc42368a

(13) Allam, N. K.; Shankar, K.; Grimes, C. A. Adv. Mater. 2008, 20, 3942. doi: 10.1002/adma.v20:20

(14) Nageh, K. A.; Karthik, S.; Grames, C. A. J. Mater. Chem. 2008, 18, 2341. doi: 10.1039/b718580d

(15) Shankar, K.; Basham, J. I.; Allam, N. K.; Varghese, O. K.; Mor, G. K.; Feng, X.; Paulose, M.; Seabold, J. A.; Choi, K. S.; Grimes, C. A. J. Phys. Chem. C 2009, 113, 6327. doi: 10.1021/ jp809385x

(16) Beranek, R.; Tsuchiya, H.; Sugishima, T.; Macak, J. M.; Taveira, L.; Fujimoto, S.; Kisch, H.; Schmuki, P. Appl. Phys. Lett. 2005, 87, 243114. doi: 10.1063/1.2140085

(17) Hirakawa, T.; Yawata, K.; Nosaka, Y. Appl. Catal. A: Gen. 2007, 325, 105. doi: 10.1016/j.apcata.2007.03.015

(18) Anbar, M.; Meyerstein, D.; Neta, P. J. Phys. Chem. 1966, 70, 2660. doi: 10.1021/j100880a034

(19) Sun, L.; Li, J.; Wang, C. L.; Lin, C. J.; Du, R. G.; Chen, H. B.
Chin. J. Inorg. Chem. 2009, 25, 334. [孙 岗, 李 静, 王成 林, 林昌健, 杜荣归, 陈鸿博. 无机化学学报, 2009, 25, 334.]

(20) Yon, S. S.; Smith, Y. R.; Misra, M.; Subramanian, V. Appl. Catal. B: Environ. 2008, 84, 372. doi: 10.1016/j.apcatb. 2008.04.021

(21) Philippidis. N.; Sotiropoulos, S.; Efstathiou, A.; Poulios, I. J. Photochem. Photobiol. A 2009, 204, 129. doi: 10.1016/j. jphotochem.2009.03.007

(22) Liu, H.; Li, X. Z.; Leng, Y. J.; Li, W. Z. J. Phys. Chem. B 2003, 107, 8988. doi: 10.1021/jp034113r

(23) Shi, J. Y.; Leng, W. H.; Cheng, X. F.; Zhang, J. Q.; Cao, C. N. Acta Phys. -Chim. Sin. 2005, 21, 971. [施晶荣, 冷文华, 程小 芳, 张鉴清, 曹楚南. 物理化学学报, 2005, 21, 971.] doi: 10.3866/PKU.WHXB20050906

(24) Liu, H.; Wu, M.; Wu, H. J.; Sun, F. X.; Zheng, Y.; Li, W. Z. Acta Phys.-Chim. Sin. 2001, 17, 286. [刘 鸿, 吴 鸣, 吴合进, 孙 福侠, 郑 云, 李文钊. 物理化学学报, 2001, 17, 286.] doi: 10.3866/PKU.WHXB20010322

(25) Liu, H.; Cheng, S.; Wu, M.; Hu, H.; Zhang, J.; Li, W.; Cao, C. J. Phys. Chem. A 2000, 104, 7016. doi: 10.1021/jp000171q

(26) Zha, Q. X. Introduction to Kinetics on Electrode Process, 3rd ed.; Science Press: Beijing, 2002; pp 386-387. [査全性. 电极 过程动力学导论. 第三版. 北京: 科学出版社, 2002: 386-387.]

(27) Leng, W. H.; Zhang, S.; Cheng, S. A.; Zhang, J. Q.; Cao, C. N. Chin. J. Chem. Phys. 2001, 14, 705. [冷文华, 张 昭, 成少 安, 张鉴清, 曹楚南. 化学物理学报, 2001, 14, 705.]

(28) Song, Y. Y.; Roy, P.; Paramasivam, I.; Schmuki, P. Angew. Chem. Int. Edit. 2010, 49, 351. doi: 10.1002/anie.200905111

(29) Berger, P.; Leitner, N. K. V.; Doré, M.; Legube, B. Water Res. 1999, 33, 433. doi: 10.1016/S0043-1354(98)00230-9

(30) Poulios, I.; Tsachpinis, I. J. Chem. Technol. Biotechnol. 1999, $74,349$.

(31) Sauer, T.; Neto, G. C.; José, H. J.; Moreira, R. F. P. M. J. Photochem. Photobiol. A 2002, 149, 147. doi: 10.1016/S10106030(02)00015-1 
Supplementary Information for Acta Phys. -Chim. Sin. 2013, 29 (11), 2392-2398 doi: 10.3866/PKU.WHXB201309043

\title{
光诱导约束刻蚀体系中羟基自由基生成的影响因素
}

\author{
胡艳 方秋艳 周剑章* 詹东平 时 康 田中群 \\ 田昭武
}

(厦门大学化学化工学院化学系, 固体表面化学国家重点实验室, 福建 厦门 361005)

\section{Factors Influencing Hydroxyl Radical Formation in a Photo-Induced Confined Etching System}

\author{
HU Yan $\quad$ FANG Qiu-Yan ZHOU Jian-Zhang* ZHAN Dong-Ping \\ SHI Kang TIAN Zhong-Qun TIAN Zhao-Wu
}

(State Key Laboratory of Physical Chemistry of Solid Surface, Department of Chemistry, College of Chemistry and Chemical Engineering, Xiamen University, Xiamen 361005, Fujian Province, P. R. China)

*Corresponding author. Email: jzzhou@xmu.edu.cn; Tel: +86-592-2189663. 


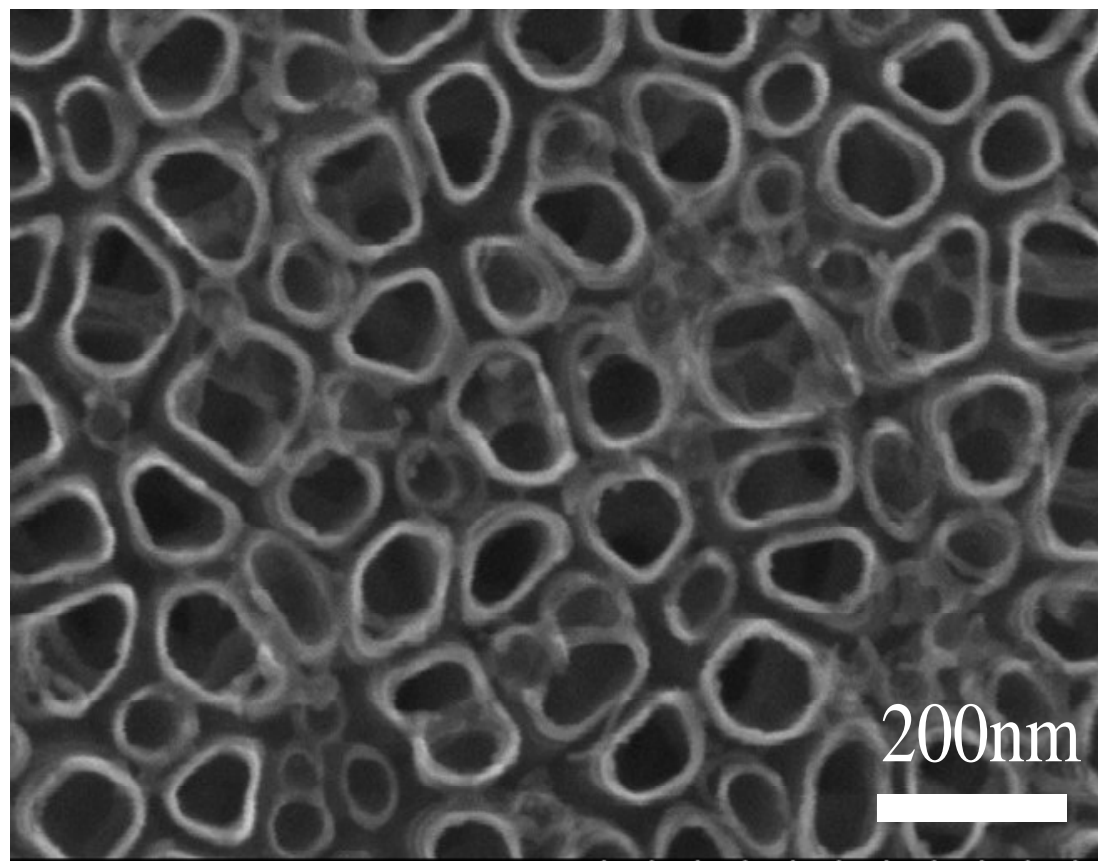

图 1 阳极氧化制备 $\mathrm{TiO}_{2}$ 纳米管阵列的 SEM 图

Fig.1 SEM image of $\mathrm{TiO}_{2}$ nanotube arrays prepared by anodization

采用阳极氧化法, 在 $\mathrm{NH}_{4} \mathrm{~F}$ 乙二醇有机体系中采用 $20 \mathrm{~V}$ 电压氧化 $30 \mathrm{~min}$. 制 备排列规整、高度有序的 $\mathrm{TiO}_{2}$ 纳米管. 所制备的 $\mathrm{TiO}_{2}$ 管径约为 $100 \mathrm{~nm}$, 壁厚约 为 $8 \mathrm{~nm}$. 阳极氧化法制备的 $\mathrm{TiO}_{2}$ 是垂直基底有序生长的, 有利于自由电子向基底 的移动, 同时纳米管的中空结构增加了 $\mathrm{TiO}_{2}$ 和溶液的接触. 与光催化常用的 $\mathrm{TiO}_{2}$ 纳米微粒膜相比, $\mathrm{TiO}_{2}$ 纳米管虽然在比表面积上不占优势，但其具有较短的 载流子扩散路径, 极大的降低光生载流子的复合, 在很大程度上弥补了其比表面 较小的不足. 


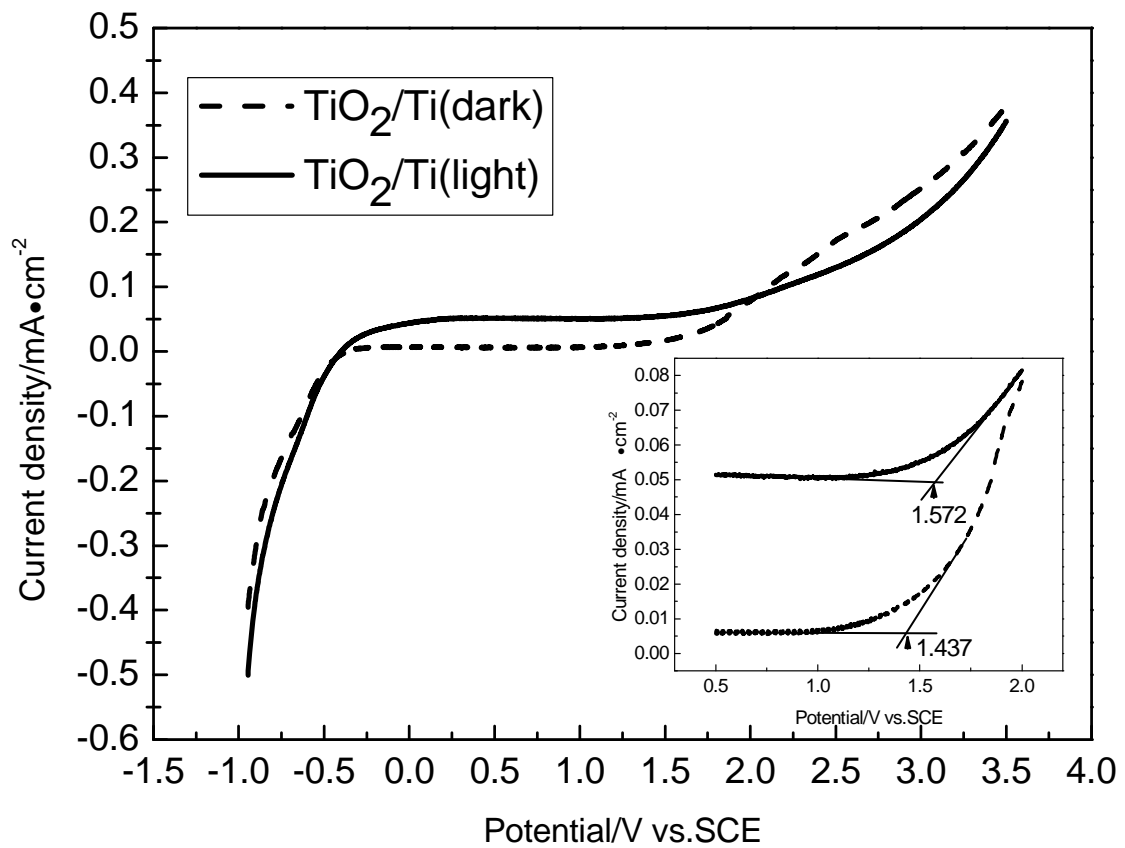

图 S2 $\mathrm{TiO}_{2}$ 纳米管电极在 $0.01 \mathrm{~mol} / \mathrm{L} \mathrm{Na}_{2} \mathrm{SO}_{4}$ 溶液中光照和暗态的线性伏安扫描 曲线

Fig.S2 Linear sweep voltammograms of $\mathrm{TiO}_{2}$ nanotube arrays electrode in 0.01 $\mathrm{mol} / \mathrm{L} \quad \mathrm{Na}_{2} \mathrm{SO}_{4}$ solution under dark and illumination condition (light intensity: $35 \mathrm{~mW} / \mathrm{cm}^{2}$ )

如图 2 所示: 当 $\mathrm{V}<-0.5 \mathrm{~V}$ 时, 随着扫描电位的正移, 电流迅速增加, 这主要 是 $\mathrm{H}_{2} \mathrm{O}$ 的还原. 在- $0.5 \mathrm{~V} \sim 1.5 \mathrm{~V}$ 之间, 电流变化很小, 呈现一种 $\mathrm{TiO}_{2}$ 钝化膜的特 性. 这是一种典型的 $\mathrm{TiO}_{2} \mathrm{n}$-型半导体特性. 当 $\mathrm{V}>1.5 \mathrm{~V}$ 时, 随着电位的正扫, 电 流迅速增加, 这主要是 $\mathrm{H}_{2} \mathrm{O}$ 的氧化. 在平坦化应用中, 所加电位应避开严重的电 极反应区域, 从 $\mathrm{I}-\mathrm{V}$ 曲线上判断, 光电催化时所加电压应在 $0 \mathrm{~V} \sim 1.5 \mathrm{~V}$ 之间较为 合适 